\title{
A new procedure for analyzing the nucleation kinetics of freezing in computer simulation
}

\author{
Lawrence S. Bartell ${ }^{\text {a) }}$ \\ Department of Chemistry, University of Michigan, Ann Arbor, Michigan 48109 \\ David T. Wu ${ }^{\text {b) }}$ \\ Department of Mechanical Engineering, Yale University, New Haven, Connecticut 06520
}

(Received 10 July 2006; accepted 21 September 2006; published online 15 November 2006)

\begin{abstract}
A new method for deriving the size of the critical nucleus and the Zeldovich factor directly from kinetic data is presented. Moreover, in principle, the form of $G(n)$, the free energy of formation of nuclei consisting of $n$ molecules, can be inferred. The method involves measuring times of first appearance of nuclei of size $n$ in the transient regime and applying the Becker-Döring theory. Times of first appearance exhibit the same characteristics as the conventional times associated with $N(n, t)$, the number of nuclei of at least size $n$ per unit volume that have materialized at time $t$. That is, they are well represented by three nucleation parameters, the reduced moment, the time lag, and the steady state nucleation rate. But unlike the conventional steady state rate which is independent of $n$, the steady state times of first appearance vary with $n$. In order to characterize the three nucleation parameters with precision, however, thousands of independent stochastic events with known $n$ are required. Such sets of data are readily generated in molecular dynamic simulations but, so far, not in laboratory experiments. Results are illustrated by an analysis of simulations of the spontaneous freezing of large clusters of $\mathrm{SeF}_{6}$. (C) 2006 American Institute of Physics. [DOI: 10.1063/1.2363382]
\end{abstract}

\section{INTRODUCTION}

Recent advances in experimental techniques and theoretical treatments have greatly improved the understanding of homogeneous nucleation in the case of the condensation of vapor. ${ }^{1-5}$ Treatments of the more difficult problem of homogeneous nucleation in freezing are at a much more primitive stage of development, however. A half century ago, Turnbull devised an experimental procedure to determine nucleation rates in freezing, free from the interference of trace amounts of impurities which might catalyze heterogeneous nucleation. ${ }^{6} \mathrm{He}$ also helped to formulate an extension of nucleation theory to treat freezing. ${ }^{7}$ His experimental technique involved the production and investigation of very finely divided liquid drops but was extremely tedious to carry out with precision. Instead, subsequent studies of freezing often investigated the crystallization of glasses. ${ }^{8-10} \mathrm{Re}-$ cently an entirely different technique was introduced by which the kinetics of freezing large liquid molecular clusters produced in supersonic flow could be studied. ${ }^{11,12}$ It avoided some of the problems associated with previous techniques but was applicable only to a limited number of substances, and its inherent depth of supercooling was very large. While all of the methods for the study of freezing were able to estimate steady state nucleation rates, none were able to supply definitive information about the nucleation process itself, namely, the size of the critical nucleus and the parameters characterizing transient nucleation.

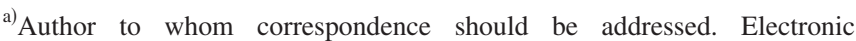
mail:lbart@umich.edu

${ }^{b)}$ Present address: Department of Chemistry, University of Michigan, Ann Arbor, MI 48109.
}

Although the study of the freezing of glasses offered the most direct way to characterize transient nucleation, in principle, analyses of the problem indicated that thousands of independent stochastic data points (sets of times at which nuclei materialize) are needed to yield a statistically reliable portrayal of the transient regime. ${ }^{13,14}$ Such large sets of data have yet to be generated in laboratory experiments. The need for such a large number of points is mainly due to the correlation between the three principal parameters to characterize the transient regime (see below). Another problem is that, since nuclei in such experiments must grow far larger than critical nuclei before they can be detected, no direct information about the size of critical nuclei can be deduced from the experimental data.

A different approach to the problem has been explored recently, that of computer experiments. ${ }^{15}$ It is possible to perform massive computer simulations of the spontaneous freezing of miniscule droplets (i.e., large liquid clusters). Such simulations can in principle provide thousands of sets of data, but spontaneous nucleation occurs too rarely to make the method practical on the time scale of computer simulations, unless the supercooling is very deep. However, the study of deep supercooling is of interest in its own right in providing information about a new regime of nucleation. One great advantage of the molecular dynamics simulations is that the time evolution of nucleation can be analyzed in molecular detail. Several techniques make it possible to distinguish between liquid regions and solidlike regions on a molecular scale. Therefore, it is feasible to determine by standard methods of analysis, with good statistical accuracy, the parameters defining the transient region. In terms of Wu's theory of moments, ${ }^{3}$ these parameters are the nucleation time 
lag $L$, the steady state rate $J_{s}$, and the "reduced moment" $M_{r}$. The point of the present paper is to show that a new method of analysis of the simulations makes it possible, in addition, to derive $n^{*}$, the size of the critical nucleus, and the Zeldovich factor directly from the kinetic data. Heretofore, no determination of these quantities has been made directly from simulations of the kinetics of spontaneous phase changes. In condensation of vapor, however, where nuclei differ considerably from the mother phase, ter Horst and Kashchiev have derived these quantities from Monte Carlo simulations of the probability of growth of nuclei of size $n .{ }^{16}$ This method has not been found to work effectively for the process of freezing, however. ${ }^{17} \mathrm{~A}$ description of the new method is presented in the next section.

\section{PROCEDURE}

Standard methods of analysis investigate $N(n, t)$, the number of nuclei of size $n$ or larger to have materialized per unit volume at time $t$. The steady state nucleation rate $J_{s}$ can be found from the slope $d N(n, t) / d t$ at large $t$, and this rate is independent of the size $n$. Extrapolating the tangent to this large- $t$ slope back to $N=0$ gives the time lag $L$ as the intercept. This time lag, of course, does depend on the value of $n$, but the quantity $n$ has not been known in conventional experimental studies. To determine the reduced moment, another quantity dependent on $n$, means to fit the experimental curve $N(n, t)$ by some model theoretical expression. We will apply Wu's expression in the following: ${ }^{3}$

$$
\begin{aligned}
N(t)= & J_{s}\left\{t\left[1-\frac{1}{2} \operatorname{erfc}\left(\frac{\ln \left(t M_{r}^{1 / 2} / L\right)}{\left[2 \ln \left(M_{r}\right)\right]^{1 / 2}}\right)\right]\right. \\
& \left.-L\left[1-\frac{1}{2} \operatorname{erfc}\left(\frac{\ln \left(t M_{r}^{-1 / 2} / L\right)}{\left[2 \ln \left(M_{r}\right)\right]^{1 / 2}}\right)\right]\right\},
\end{aligned}
$$

where $M_{r}$ is the reduced moment. This expression was used in the derivation of nucleation rates from the simulations. In the case of molecular dynamics simulations resulting in the formation of nuclei of size $n$ in a set of $N_{0}$ independent clusters, it is appropriate to delete a cluster from the set once it has nucleated, leaving $N_{c}(t)<N_{0}$ clusters as candidates for forming nuclei of size $n .{ }^{18}$ Therefore, the volume $V$ available for further nucleation is

$$
V=N_{c} \times V_{c},
$$

where $V_{c}$ is the volume per cluster. To obtain an expression for clusters analogous to Eq. (1), the variable $-\ln \left[N_{c}(t) / N_{0}\right] / V_{c}$ is substituted for $N(t)$.

It should be mentioned that according to the BeckerDöring theory, ${ }^{19}$ in the case of subcritical nuclei, $J(n, t)$ may overshoot the steady state rate at small times, rendering Eq. (1) inapplicable. In such cases, of course, the transient regime is no longer adequately characterized by the aforementioned three parameters.

According to the new method of analysis, instead of finding the set of times $t$ at which $N(n, t)$ nucleation events have taken place, the set of times $t^{\mathrm{FA}}(n)$ of the first appearance of nuclei of size $n$ is determined over a range of sizes. The classical expression for the free energy of formation of a nucleus of size $n$ involves a volume plus a surface term, ${ }^{20}$ or setting the reference free energy of the monomer to zero,

$$
G(n)=-A(n-1)+B\left(n^{2 / 3}-1\right),
$$

where $A$ pertains to the free energy lowering for freezing per unit volume and $B$ is related to the free energy cost of forming the interface between the solid nucleus and the surrounding liquid. If this expression is adopted, then the BeckerDöring theory ${ }^{19}$ yields an explicit expression for the nucleation rate. The following development outlines the treatment and the modification needed to arrive at the new method of analysis. Consider the addition of a monomer $A_{1}$ to an $(n-1)$-mer $A_{n-1}$,

$$
A_{1}+A_{n-1} \underset{\alpha_{n}}{\stackrel{\beta_{n-1}}{\rightleftarrows}} A_{n} .
$$

The net rate of conversion can be expressed in terms of rate constants $\alpha_{n}$ and $\beta_{n-1}$ and populations $f_{n}$ of $n$-mers per unit volume as

$$
J_{n}=\beta_{n-1} f_{n-1}-\alpha_{n} f_{n} .
$$

Invoking the constrained equilibrium hypothesis, the BeckerDöring theory gives the expression for the steady state nucleation rate,

$$
J_{s}=\frac{f_{1}}{\sum_{n=1}^{n_{\max }-1} 1 / \beta_{n} e^{-G(n) / k T}}=\frac{f_{1} e^{-G^{*} / k T}}{\sum_{n=1}^{n_{\max }-1} e^{\left[G(n)-G^{*}\right] / k T} / \beta_{n}},
$$

where $G^{*}$ is the free energy barrier to the formation of the critical nucleus. The Zeldovich approximation for the evaluation of Eq. (6) is

$$
J_{s}=\beta^{*} f_{1} e^{-G^{*} / k T} / \int_{-\infty}^{\infty} d\left(n-n^{*}\right) e^{(1 / 2 k T)\left(\partial^{2} G(n) / \partial n^{2}\right)_{n}{ }^{*}\left(n-n^{*}\right)^{2}}
$$

so that the steady state nucleation rate becomes

$$
J_{s}=\beta^{*} f_{1} Z e^{-G^{*} / k T},
$$

where $Z$ is the Zeldovich factor

$$
Z=\left[\frac{-1}{2 \pi k T}\left(\frac{\partial^{2} G(n)}{\partial n^{2}}\right)_{n=n^{*}}\right]^{1 / 2} .
$$

How this relates to the analysis of times of first appearance $t^{\mathrm{FA}}(n)$ of nuclei the size of $n$ can be understood from the fact that the difference between the foregoing treatment and that based on times of first appearance is that at $t^{\mathrm{FA}}(n)$, no nuclei larger than $n$ have yet appeared. Therefore, the upper limit in the sum in Eq. (6) becomes $(n-1)$, making the upper limit in the integral of Eq. (7) equal to $n$. Carrying out the modified integration and comparing the result with that of Eq. (7) yields

$$
\frac{J_{s}}{J_{s}^{\mathrm{FA}}}=\frac{1}{2} \operatorname{erfc}\left[\sqrt{\pi} Z\left(n^{*}-n\right)\right]
$$

which has the value of $\frac{1}{2}$ when $n$ is the size of the critical nucleus. Therefore, a plot of $J_{s} / J_{s}^{\mathrm{FA}}$ reveals the size of the 


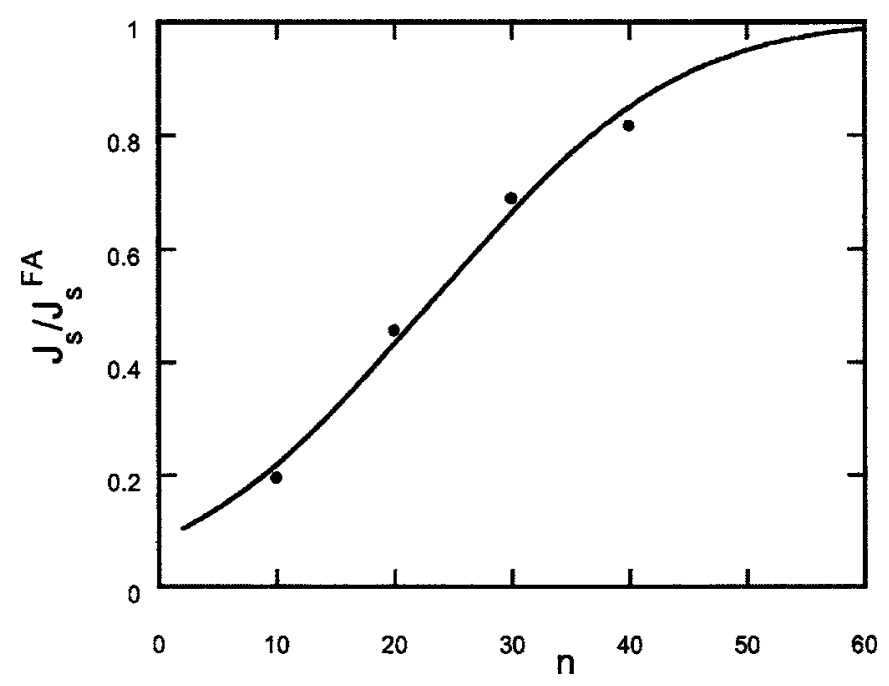

FIG. 1. MD values of the ratio of the conventional steady state rate to the steady state rate for times of first appearance. Curve: the expected ratio according to Eq. (10).

critical nucleus as well as the value of the Zeldodvich factor for those systems freezing in accordance with the BeckerDöring-Zeldovich theory. A somewhat more accurate expression can be made by replacing the infinite lower limit in the integral of Eq. (7) by $n=1$.

\section{RESULTS}

It turns out that a plot of the ordered set of times of first appearance against $-\ln \left(N_{c}(t) / N_{0}\right)$ found for a set of $N_{0}$ nucleation events has the same characteristic appearance as a plot of the set of times of formation of $N(n, t)$ nuclei per unit time. ${ }^{15}$ Each plot exhibits a transient regime followed by a steady state regime. Therefore, it is straightforward to derive the steady state rate $J_{s}^{\mathrm{FA}}$ of first appearances of nuclei of size $n$. The difference between the steady state rates, of course, is that the rates for times of first appearance depend on $n$, whereas the conventional steady state rate $J_{s}$ is independent of $n$. In Fig. 1 the ratios of steady state values $J_{s} / J_{s}^{\mathrm{FA}}(n)$ versus $n$ as derived elsewhere are shown for a set of 2500 freezing events in liquid clusters containing $\sim 545$ molecules of $\mathrm{SeF}_{6}$. According to Eq. (10), the size of the critical nucleus corresponds to $n$ at which $J_{s} / J_{s}^{\mathrm{FA}}(n)$ is equal to $\frac{1}{2}$, namely, $\approx 23$ molecules, and the Zeldovich factor to fit the shape of $J_{s} / J_{s}^{\mathrm{FA}}(n)$ is $\approx 0.024$. In principle, it is possible also to derive the nucleation barrier $G^{*} / k T$ by invoking Eq. (3) and applying Eq. (9). A value for $G^{*}$ of about $6 k T$ is found, but in view of the preliminary nature of the data and treatment, the accuracy cannot be expected to be very high.

\section{DISCUSSION}

Among the advantages of the new method of analysis are the information it yields about the size of the critical nucleus and the Zeldovich factor. In this respect, though not in the kind of information it is based on, it bears a resemblance to the "kinetic nucleation theorem" treat nucleation in condensation. This theorem yields $n^{*}$ and also, via the second cumulant, $Z$.
Results illustrated in Fig. 1 are preliminary in that the original simulations were carried out before the idea of determining times of first appearance had been conceived. Intervals between saved configurations were sufficiently large that the times of nucleation for small nuclei (containing an integral number of molecules) had to be estimated by an interpolation procedure of limited precision. Even if the precision is somewhat crude, the new procedure for estimating the size of critical nuclei appears to be promising. It should be pointed out, however, that the size depends on the order parameter applied to identify which molecules belong to the solidlike region and which to the surrounding liquid. This same problem is seen in computations of critical nuclei derived in density functional computations. ${ }^{22}$ If the translational order parameter is used (the $Q_{6}$ parameter in the present paper), the size is larger than if the local density is used. Here we remark that in molecular dynamics (MD) simulations, unfortunately, the local density is a parameter too noisy to yield very accurate results. But the differences between results when density, molecular orientational order, $^{23}$ and translational order parameters are used have a simple physical interpretation.

As Turnbull predicted many years ago, ${ }^{24}$ when a crystalline nucleus is surrounded by liquid, the liquid molecules tend to conform to the crystal surface as closely as possible, leading to an enhanced order imposed on the liquid at the interface, and this accounts for the negative excess interfacial entropy Turnbull measured in his experiments. The local density order parameter corresponds most nearly to the crystalline nucleus itself, while the $Q_{6}$ parameter includes the ordered liquid surrounding the nucleus. The molecular orientational order parameter was found to correlate closely with the local density parameter. It is a matter of taste for the interpreter to decide what is the true nucleus. It turns out that the $Q_{6}$ result for $n^{*}$ is not far from the value given by the classical theory of nucleation plus a layer of molecules surrounding the classical nucleus. This result is interesting but not profound because the classical theory itself, neglecting the thickness of interfaces and invoking bulk thermodynamic properties to regions containing only a few molecules, is not rigorous.

Another aspect of the analysis may be significant. The Zeldovich approximation in the foregoing invoked the classical expression (3) for the free energy of forming a nucleus of size $n$. It is this expression for $G(n)$ which gives rise to the erfc function in Eq. (10). If an expression different from (3) had been adopted in the sum of Eq. (6), and the corresponding integral had been evaluated accurately, a different shape of the curve $J_{s} / J_{s}^{\mathrm{FA}}(n)$ versus $n$ would have resulted. It is obvious that Eq. (3), the classical expression, cannot be rigorously correct for small $n$. It is plausible that the small size of nuclei together with effects of interfacial thickness tend to make coefficients $A$ and $B$ size dependent and probably smaller for nuclei than for the bulk. Therefore, it is possible that accurate measurements of $J_{s} / J_{s}^{\mathrm{FA}}(n)$ might yield not only the size of critical nuclei but also an improved form for $G(n)$. In any event, the preliminary plot of $J_{s} / J_{s}^{\mathrm{FA}}(n)$ versus $n$ does not conform accurately to the form of Eq. (10). How much the misfit is due to the coarse interpolations to find the set of 
$t^{\mathrm{FA}}(n)$ and how much it is an indication of the deviation of Eq. (3) from a physically more accurate expression are so far unknown.

\section{CONCLUDING REMARKS}

Only computer experiments to date have been able to generate a sufficient number of nucleation events of known nuclear size to establish, with statistical accuracy, values of the parameters characterizing the transient regime. But that is not the only virtue of the computer simulations. The new technique of analyzing data from simulations provides a set of times of first appearance of nuclei of size $n$. These can reveal the size of critical nuclei, a parameter that has been difficult to establish in past studies, as well as a value for the Zeldovich factor. An additional potentiality of the simulations has been suggested, namely, that they may also yield a physically more accurate determination of the form of the free energy of nucleation than that given by the classical expression for $G(n)$. An examination of how effective the new approach to analysis can be in supplying the information latent within the data generated in simulations is under investigation.

\footnotetext{
${ }^{1}$ K. F. Kelton, A. L. Greer, and C. V. Thompson, J. Chem. Phys. 79, 6261 (1983).

${ }^{2}$ A. Laaksonen, V. Talanquer, and D. W. Oxtoby, Annu. Rev. Phys. Chem.
}

46, 489 (1995).

${ }^{3}$ D. T. Wu, in Solid State Physics, edited by H. Ehrenreich and F. Spaepen (Academic, New York, 1997), Vol. 50, p. 37.

${ }^{4}$ D. Kashchiev, Nucleation: Basic Theory with Applications (ButterworthHeinemann, Oxford, 2000).

${ }^{5}$ V. A. Shneidman, J. Chem. Phys. 119, 13487 (2003).

${ }^{6}$ D. Turnbull, J. Chem. Phys. 18, 198 (1950).

${ }^{7}$ D. Turnbull and J. C. Fisher, J. Chem. Phys. 17, 71 (1949).

${ }^{8}$ V. N. Filipovich and A. M. Kalinina, Izv. Akad. Nauk SSSR, Neorg. Mater. 7, 1844 (1971); Inorg. Mater. 7, 1645 (1971).

${ }^{9}$ A. M. Kalinina, V. N. Filipovich, and V. M. Fokin, J. Non-Cryst. Solids 38, 47 (1980)

${ }^{10}$ K. F. Kelton, in Solid State Physics, edited by H. Ehrenreich and D. Turnbull (Academic, New York, 1991), Vol. 45, p. 75.

${ }^{11}$ T. S. Dibble and L. S. Bartell, J. Phys. Chem. 96, 8603 (1992).

${ }^{12}$ L. S. Bartell, Annu. Rev. Phys. Chem. 49, 43 (1998).

${ }^{13}$ L. S. Bartell, J. Phys. Chem. A 106, 10893 (2002).

${ }^{14}$ E. J. Jacob and L. S. Bartell, J. Phys. Chem. A 107, 1859 (2003).

${ }^{15}$ G. W. Turner and L. S. Bartell, J. Phys. Chem. B 108, 19742 (2004).

${ }^{16}$ J. H. ter Horst and D. Kashchiev, J. Chem. Phys. 119, 2241 (2003).

${ }^{17}$ G. W. Turner and L. S. Bartell (unpublished).

${ }^{18} \mathrm{D}$. W. Oxtoby (private communication).

${ }^{19}$ R. Becker and W. Döring, Ann. Phys. 24, 719 (1935).

${ }^{20}$ J. W. Gibbs, The Scientific Papers of J. Willard Gibbs (Dover, New York, 1961).

${ }^{21}$ R. McGraw and D. T. Wu, J. Chem. Phys. 118, 0337 (2003).

${ }^{22}$ P. Harrowell and D. W. Oxtoby, J. Chem. Phys. 80, 1639 (1984).

${ }^{23}$ Y. G. Chushak and L. S. Bartell, J. Phys. Chem. A 104, 9328 (2000).

${ }^{24} \mathrm{D}$. Turnbull, in Proceedings of the 1964 International Conference on Physics of Non-Crystalline Solids, edited by J. A. Prins (North-Holland, Amsterdam, 1964), p. 4. 